\title{
Portal vein thrombosis relevance on liver cirrhosis: Italian Venous Thrombotic Events Registry
}

\author{
Francesco Violi ${ }^{1}$ Roberto Gino Corazza ${ }^{2} \cdot$ Stephen Hugh Caldwell $^{3} \cdot$ \\ Francesco Perticone $^{4} \cdot$ Angelo Gatta $^{5} \cdot$ Mario Angelico $^{6} \cdot$ Alessio Farcomeni $^{7}$. \\ Michela Masotti $^{2} \cdot$ Laura Napoleone $^{1} \cdot$ Annarita Vestri $^{7} \cdot$ Valeria Raparelli $^{1}$. \\ Stefania Basili ${ }^{1} \cdot$ PRO-LIVER Collaborators
}

Received: 18 December 2015/Accepted: 17 February 2016/Published online: 30 March 2016 (C) SIMI 2016

\begin{abstract}
Portal vein thrombosis may occur in cirrhosis; nevertheless, its prevalence, and predictors are still elusive. To investigate this issue, the Italian Society of Internal Medicine undertook the "Portal vein thrombosis Relevance On Liver cirrhosis: Italian Venous thrombotic Events Registry" (PRO-LIVER). This prospective multicenter study includes consecutive cirrhotic patients undergoing Doppler ultrasound examination of the portal area to evaluate the prevalence and incidence of portal vein thrombosis over a 2-year scheduled follow-up. Seven hundred and fifty-three (68\% men; $64 \pm 12$ years)
\end{abstract}

The members of PRO-LIVER Collaborators are listed in Appendix section.

Electronic supplementary material The online version of this article (doi:10.1007/s11739-016-1416-8) contains supplementary material, which is available to authorized users.

Francesco Violi

francesco.violi@uniroma1.it

1 I Clinica Medica, Department of Internal Medicine and Medical Specialties, Sapienza-University of Rome, Viale del Policlinico 155, 00161 Rome, Italy

2 First Department of Internal Medicine, Fondazione IRCCS Policlinico San Matteo, University of Pavia, Pavia, Italy

3 Division of Gastroenterology and Hepatology, Digestive Health Center, University of Virginia, Charlottesville, VA, USA

4 Department of Medical and Surgical Sciences, University of Catanzaro, Catanzaro, Italy

5 Department of Medicine, University of Padova, Padua, Italy

6 Liver Unit, University Hospital Tor Vergata, Rome, Italy

7 Department of Public Health and Infectious Disease, Sapienza-University of Rome, Rome, Italy patients were included in the present analysis. Fifty percent of the cases were cirrhotic outpatients. Viral (44\%) etiology was predominant. Around half of the patients had a mild-severity disease according to the Child-Pugh score; hepatocellular carcinoma was present in $20 \%$. The prevalence of ultrasound-detected portal vein thrombosis was $17 \%(n=126)$; it was asymptomatic in $43 \%$ of the cases. Notably, more than half of the portal vein thrombosis patients $(n=81)$ were not treated with anticoagulant therapy. Logistic step-forward multivariate analysis demonstrated that previous portal vein thrombosis $(p<0.001)$, Child-Pugh Class B $+\mathrm{C}(p<0.001)$, hepatocellular carcinoma $(p=0.01)$, previous upper gastrointestinal bleeding $(p=0.030)$ and older age $(p=0.012)$ were independently associated with portal vein thrombosis. Portal vein thrombosis is a frequent complication of cirrhosis, particularly in patients with moderate-severe liver failure. The apparent undertreatment of patients with portal vein thrombosis is a matter of concern and debate, which should be addressed by planning interventional trials especially with newer oral anticoagulants.

Clinicaltrials.gov identifier NCT01470547.

Keywords Splanchnic venous thrombosis .

Anticoagulants - Liver failure - Hepatocellular carcinoma . Esophageal varices

\section{Introduction}

For decades, cirrhosis patients have been considered at risk of bleeding complications, which were believed to stem from impaired clotting activation coincidentally with deterioration of liver function. However, the term "coagulopathy", which has been coined to indicate the 
association between clotting changes and bleeding [1], has been recently challenged because, apart from gastrointestinal (GI) variceal bleeding, which is generally unrelated to clotting changes, bleeding complications in cirrhosis are rare [2]. Conversely, a large body of evidences has been accumulating to indicate an association between thrombosis and cirrhosis, particularly in patients with decompensated disease [3]; thrombosis may occur in the portal vein (portal vein thrombosis, PVT) or systemic circulation [4-7].

Cirrhosis is the underlying cause of PVT in $22-28 \%$ of all cases [3]. The prevalence of PVT in cirrhosis is variable depending on the diagnostic procedure and on the degree of liver failure. In angiography or surgery studies, the prevalence of PVT ranges from 0.6 to $16 \%$; using ultrasonography, the reported prevalence is as high as 10-25\% [3]. The prevalence of PVT also increases with the severity of cirrhosis, being approximately $1 \%$ in patients with compensated cirrhosis and rising to $8-25 \%$ in candidates for liver transplantation [3]. Important limitations of most past studies of PVT in cirrhosis include their retrospective design and the small sample size, making it difficult to draw firm conclusions. Furthermore, if detection is based solely on the presence of overt symptoms, PVT may be underestimated, as it is often first detected in asymptomatic patients. Its significance in this setting remains a point of debate, but can be better understood with complete and more definitive knowledge of its prevalence and incidence.

Clinical and laboratory predictors of PVT are also still unclear. Among the local factors, decreased portal flow velocity and coexistent hepatocellular carcinoma (HCC) were frequently observed [8-10]. Recently, in a post hoc analysis of THROMBOCIR study, among 1243 ChildPugh A and B cirrhotic patients, the baseline risk factors independently associated with PVT were esophageal varices and prothrombin time (PT) [11]; however, the predictors of PVT in the real world of cirrhosis including a wider range of liver failure severity are still elusive.

To further study the PVT prevalence and risk factors related to it, the Italian Society of Internal Medicine (SIMI) designed a registry exploring PVT prevalence in the day to day world of cirrhosis management and included patients with different degrees of liver failure as classified by Child-Pugh or MELD score [12, 13]. The "Portal vein thrombosis Relevance On Liver cirrhosis: Italian Venous thrombotic Events Registry" (PRO-LIVER) study started in January 2012. The specific aim of the present analysis from the PRO-LIVER registry was to estimate the prevalence of PVT, as detected by upper abdominal Doppler ultrasonography (US) examination and to depict the clinical independent factors associated with PVT presence in cirrhosis.

\section{Methods}

\section{Study design}

The PRO-LIVER study is an ongoing Italian-based prospective multicenter study with the primary objective of estimating the prevalence of PVT in a cohort of patients with cirrhosis of any etiology and severity.

As secondary end points, it was planned that the yearly evaluation over the 2-year follow-up would include the following events: (1) venous thrombotic events [i.e., deep vein thrombosis/pulmonary embolism (VTE) and PVT]; (2) bleeding events (i.e., GI or non-GI hemorrhage); (3) overall mortality; (4) hospital admissions for decompensated cirrhosis and (5) occurrence of cirrhosis-related complications (i.e., onset or progression of esophageal varices, ascites or refractory ascites, jaundice, onset of liver cancer, infections, spontaneous bacterial peritonitis, onset of hepato-renal or hepatic-pulmonary syndrome).

The SIMI coordinates all regional centers (see Online Appendix 1) involved in the study, having the same standard of care, by the creation of a network for the recruitment and monitoring of cirrhotic patients.

This study was conducted in accordance with the EU Note for Guidance on Good Clinical Practice CPMP/ECH/ 135/95 and the Declaration of Helsinki. Informed consent was obtained from all individual participants included in the study. The study was initiated only after local and ethic approval requirements were obtained (ClinicalTrials.gov Identifier: NCT01470547). The center's participation in the registry was voluntary and not sponsored.

\section{Study population}

All consecutive cirrhotic patients who were referred to the 43 participating centers $(n=33$ internal medicine units; $n=10$ hepatology units) were enrolled. The presence of concomitant extra-hepatic neoplasms was the only exclusion criteria. Thus, we included patients with a diagnosis of cirrhosis of any etiology and severity (including cirrhosis complicated by HCC).

At baseline, complete medical history, thrombosis risk factor evaluation, anthropometric data and evaluation of the severity of cirrhosis were registered. The Child-Pugh score [12] and MELD score [13] were assessed to establish the severity of liver disease. In addition, the state of liver disease compensation was reported according to Baveno IV score [14].

Among laboratory variables, only prothrombin time, total bilirubin, serum albumin and serum creatinine were mandatory to allow the Child-Pugh and MELD calculation. However, additional laboratory parameters could be inserted in the standard form at discretion of the investigator. 


\section{Doppler ultrasound examination}

A Doppler US examination of the portal vein main trunk and its branches and tributaries was mandatory to evaluate the presence of PVT. Standard US parameters that were assessed included the presence or absence of focal liver lesions, the spleen diameter and a complete evaluation of the portal vein axis and were reported on a standardized form (see Online Appendix 2). If available, portal vein flow velocity was also recorded.

\section{Portal vein thrombosis: definition}

By pre-set study criteria, PVT was first suspected when solid endoluminal material was detected in the main trunk of the portal vein and/or its branches, and it was confirmed by demonstration of a filling defect on Doppler examination. Occlusive/complete PVT was defined by a thrombus leaving no channel for blood flow. Otherwise, PVT was considered to be non-occlusive/incomplete. The definition of previous PVT was reported by investigators as a positive clinical history of PVT. For all patients with previous PVT, we requested to provide instrumental information to support this previous event (i.e., instrumental demonstration of PVT resolution) and to validate PVT recurrence.

\section{Data collection and validation}

In each center, data were collected using an electronic case report form (CRF: http://www.simi.it/attivita/ricerca/PRO LIVER/). Data were transferred to the web-central database (Coordination Center-I Clinica Medica, Sapienza-University of Rome). Using a validation plan integrated in the data entry software, data were checked for missing or contradictory entries and values out of the normal range. A final database was created and validated by the study coordinators (see Online Appendix 1). Patient's identification name was registered in the participating centers, but was not transferred to the central database. Patients were identified by a serial number for each center.

\section{Sample size determination}

We originally planned to include in the study $n=1100$ patients. After 36 months of enrollment, the Data and Safety Monitoring Board (see Online Appendix 1) decided to terminate the trial due to insufficient accrual rate. This decision was taken before any data disclosure and therefore had no impact on the estimates other than a slightly larger expected confidence interval. The trial was terminated, considering that the current sample size would guarantee, assuming (as originally planned) an expected prevalence of $18 \%$ at time zero, a $95 \%$ confidence interval with a width less than or equal to $5.3 \%$. This width was deemed to be satisfactory and the trial was terminated.

\section{Statistical analysis}

All continuous variables were tested for normality with the Shapiro-Wilk test. Variables with normal distribution were expressed as mean and standard deviation and tested for differences with the Student's $t$ test. Non-normal variables were expressed as median and interquartile range (IQR) and differences tested with the Mann-Whitney $U$ test. Categorical variables were expressed as counts and percentages and analyzed by a Chi-square test.

A logistic regression analysis was performed to establish all clinical factors significantly associated with PVT presence. All variables entered the multivariate logistic model; a forward stepwise method was used to build the final model. A two-sided $p$ value $<0.05$ was considered to be statistically significant. All analyses were performed using SPSS v. 22.0 (IBM, NY, USA).

\section{Results}

From January 2012 to December 2014, among 43 enrolling units, a total of 802 consecutive cirrhotic patients were enrolled. Two participant centers, which globally recruited 49 patients, were excluded from the analysis for a selection bias, as they enrolled only patients with PVT. Therefore, 753 consecutive cirrhotic patients were included in the present analysis (Fig. 1). Approximately, $50 \%$ of the cases were outpatients.

\section{Patients}

The overall mean age was $64 \pm 12$ years and $68 \%$ were men. Viral $(44 \%)$ or alcoholic $(25 \%)$ etiologies were predominant. Around half of the patients $(47 \%)$ had a

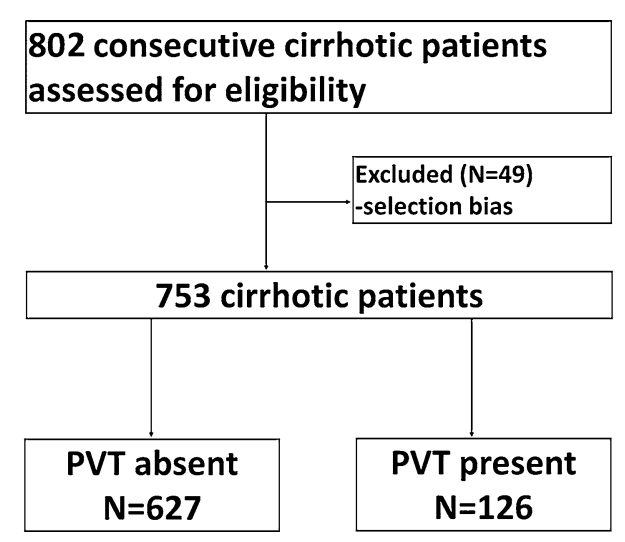

Fig. 1 Flow chart of the study. $P V T$ portal vein thrombosis 
moderate-severe disease according to Child-Pugh score (i.e., classes B or C) and similarly compensated cirrhosis was evident in over half $(57 \%)$ according to the Baveno IV score. HCC was detected in $20 \%$. Seventeen patients had an inserted transjugular intrahepatic portosystemic shunt prior to study entry.

PVT was detected in 126 patients (17\%), with occlusion only in the main trunk or its branches in 81 patients (64\%), while obstruction of more than one portal vein branches was present in 45 patients (36\%); an extension of thrombosis to the mesenteric-splenic veins was reported in 27 patients $(21 \%)$. According to Yerdel grade [15], PVT in our cohort was classified as follows: $60 \%$ grade I, $19 \%$ grade II and $21 \%$ grade III-IV. A non-occlusive/incomplete PVT was present in $95 \%$ of the cases, independent of the site of thrombosis. In the $40 \%$ of patients with USdetected PVT $(n=49)$, CT or MRI was also performed to confirm the diagnosis.

PVT was asymptomatic in 54 patients (43\%). The clinical manifestations of PVT was in $51 \%$ of the cases ascites not responsive to diuretics requiring paracentesis, in 5 patients $(4 \%)$ an upper gastrointestinal bleeding and in 2 patients an episode of acute encephalopathy ( $2 \%)$.

\section{Comparison of PVT with non-PVT}

Clinical and laboratory characteristics of patients according to the presence or absence of PVT are depicted in Table 1. Cirrhotic patients with PVT were older $(67 \pm 11$ years) and more frequently inpatients (59\%) as opposed to the outpatient setting (41\%). No differences in etiology, sex and body mass index were observed. Cirrhotic patients with PVT showed a more advanced and decompensated disease with higher prevalence of Child-Pugh B and C classes $(p<0.0037)$. Furthermore, the presence of ascites and encephalopathy, as well as diuretic treatment was more frequently observed in PVT patients.

Detection of esophageal varices of grade $\geq 2(39 \%)$, as well as the presence of indirect US signs of portal hypertension, such as increases of portal vein diameter and splenomegaly, characterized PVT patients. Compared with patients without PVT, PVT patients had an increased bipolar spleen diameter $(15 \pm 3$ vs. $14 \pm 3, p=0.0072)$.

HCC prevalence was $35 \%$ in patients with PVT versus $17 \%$ in those without PVT. PVT patients had a clinical history more complicated by previous PVT (20\%, $p<0.0001)$ and upper GI bleeding $(24 \%, p=0.0075)$. Among the laboratory parameters, only serum albumin and platelet count differed between patients with and without PVT (Table 1).

Eighty-one out of 126 PVT patients (64\%) did not receive any anticoagulant treatment; among PVT on treatment with anticoagulants, 33 were being treated with low molecular weight heparins, 7 with fondaparinux and 5 with warfarin.

Table 2, panel A, reported variables significantly associated with PVT on univariate analysis. All these variables entered the multivariate logistic analysis. The final model (Table 2, panel b) showed that previous PVT $(p<0.001)$, Child-Pugh class B $+\mathrm{C}(p<0.001)$, HCC $(p=0.01)$, previous upper GI bleeding $(p=0.030)$ and older age $(p=0.012)$ were significantly associated with the presence of PVT.

\section{Discussion}

In this large multicenter study, we demonstrate that PVT is a frequent complication of cirrhosis with about one-fifth of patients suffering from this vascular complication; older patients with more severe liver failure were at higher risk of PVT.

The present study supports and extends previous findings on this topic indicating that thrombosis may frequently occur in cirrhosis whatever is its etiology. This complication is biologically plausible, as previously shown by the demonstration of an ongoing prothrombotic state in the portal vein of cirrhotic patients undergoing transjugular intrahepatic portosystemic shunt [16]; of note, markers of clotting activation were also detected in the peripheral circulation of cirrhotic patients compared to controls [17]. Among the mechanisms potentially accounting for PVT, there is experimental and clinical evidence that bacterial endotoxins such as lipopolysaccharide (LPS) may predispose to thrombosis [18]. Thus, patients with cirrhosis show an increased concentration of bacterial endotoxins in the portal and systemic circulation compared to controls [16, 17]. This "low-grade" endotoxemia is related to translocation of bacteria and bacteria products (such as endotoxins from intestinal lumen to the portal circulation) and to endotoxin spillover into systemic circulation [19]. Together, these data led us to hypothesize that low-grade endotoxemia might favor thrombosis; in support of this, experimental and clinical studies demonstrated that in cirrhosis, endotoxemia affects Virchow's triad, i.e., hypercoagulation, endothelial damage and reduced flow velocity, which are crucial for thrombus formation $[18,20$, 21].

Among the factors associated with PVT, age and liver failure seem to have a prominent role; thus, older patients and those with moderate-severe liver failure are those in whom PVT is more prevalent. This more frequent association may be explained by the fact that the ongoing prothrombotic state is more frequent in patients of Child-Pugh classes B and C compared to class A [15, 22, 23]. Another novel finding of the study is that patients with PVT have a 
Table 1 Distribution variables according to the presence of PVT

\begin{tabular}{llll}
\hline Variables & $\begin{array}{l}\text { Patients without PVT } \\
(n=627)\end{array}$ & $\begin{array}{l}\text { Patients with PVT } \\
(n=126)\end{array}$ & $p$ \\
\hline Age (mean $\pm \mathrm{SD})$ & $64 \pm 12$ & $67 \pm 11$ & 0.0047 \\
Male sex, $n(\%)$ & $432(69)$ & $81(64)$ & 0.3105 \\
BMI $\left(\mathrm{kg} / \mathrm{m}^{2}\right)$ & $26 \pm 4$ & $26 \pm 5$ & 0.2746 \\
Inpatients, $n(\%)$ & $283(45)$ & $75(59)$ & 0.0031 \\
Etiology & & & 0.6681 \\
Alcohol, $n(\%)$ & $149(24)$ & $37(29)$ & \\
Viral, $n(\%)$ & $274(44)$ & $58(46)$ & \\
NASH/metabolic, $n(\%)$ & $38(6)$ & $5(4)$ & \\
Autoimmune, $n(\%)$ & $15(2)$ & $3(2)$ & \\
Mixed, $n(\%)$ & $89(14)$ & $13(10)$ & \\
Others/unknown, $n(\%)$ & $62(10)$ &
\end{tabular}

Child-Pugh Score

Class A, $n(\%)$

$352(56)$

45 (37)

Class B, $n(\%)$

194 (31)

$64(50)$

Class C, $n(\%)$

81 (13)

17 (13)

7 [6-9]

0.1129

Child-Pugh Score, median [IQR]

6 [5-8]

12 [10-14]

0.0216

Baveno Score

Compensated, $n(\%)$

387 (62)

0.0004

Decompensated, $n(\%)$

Previous thrombotic events

Previous portal vein thrombosis, $n(\%) \quad 26(4)$

$12(2)$

$26(20)$

$<0.0001$

Previous VTE, $n(\%)$

4 (3)

0.8451

Bleeding GI events

Upper GI bleeding, $n(\%) \quad 33(5)$

5 (4)

0.5537

Previous upper GI bleeding, $n(\%)^{\mathrm{a}}$

89 (15)

30 (24)

0.0075

Lower GI bleeding, $n(\%)$

$10(2)$

4 (3)

0.2309

Previous lower GI bleeding, $n(\%)^{\mathrm{b}}$

$33(5)$

8 (6)

0.6060

Esophageal varices, $n(\%)^{\mathrm{c}}$

$$
\text { NO, } n(\%)
$$

200 (42)

177 (37)

85 (18)

17 (3)

0.00018

Ascites

$$
\text { NO, } n(\%)
$$

407 (65)

164 (26)

56 (9)

25 (24)

39 (37)

29 (28)

11 (11)

Responsive, $n(\%)$

Refractory, $n(\%)$

Encephalopathy

$$
\text { NO, } n(\%)
$$

540 (86)

Mild, $n(\%)$

Moderate-severe, $n(\%)$

77 (12)

$10(2)$

108 (17)

$3.4 \pm 0.6$

$2.0 \pm 2.8$

$1.29 \pm 0.34$

$0.95 \pm 0.65$

$116 \pm 68$

57 (45)

$53(42)$

16 (13)

96 (76)

27 (21)

3 (2)

44 (35)

$<0.0001$

$3.1 \pm 0.6$

$<0.0001$

$2.6 \pm 4.3$

0.0508

$1.33 \pm 0.23 \quad 0.3040$

$0.97 \pm 0.39 \quad 0.7450$

Creatinine $(\mathrm{mg} / \mathrm{dL})$

$103 \pm 58$

0.0491

, $n)$

Aldosterone receptor antagonist

$330(53)$

$80(63)$

0.0255 
Table 1 continued

\begin{tabular}{llll}
\hline Variables & $\begin{array}{l}\text { Patients without PVT } \\
(n=627)\end{array}$ & $\begin{array}{l}\text { Patients with PVT } \\
(n=126)\end{array}$ & $p$ \\
\hline Loop diuretics, $n(\%)$ & $315(50)$ & $79(63)$ & 0.0106 \\
Beta-blockers, $n(\%)$ & $277(51)$ & $67(59)$ & 0.1412 \\
\hline
\end{tabular}

${ }^{\text {a }}$ Data recorded in 731 patients

b Data recorded in 741 patients

c Data recorded in 583 patients

${ }^{d}$ Data available in 753 patients

Table 2 Univariate analysis (Panel A) and logistic multivariate analysis (final model, Panel B)

\begin{tabular}{lccc}
\hline Variables & OR & $95 \%$ CI & $p$ values \\
\hline Panel A & & & \\
Age & 1.02 & $1.00-1.04$ & 0.005 \\
Inpatients & 1.78 & $1.21-2.64$ & 0.003 \\
Child-Pugh B + C & 2.21 & $1.49-3.28$ & $<0.0001$ \\
Decompensated cirrhosis & 2.43 & $1.64-3.60$ & $<0.0001$ \\
Ascites & 2.23 & $1.52-3.29$ & $<0.0001$ \\
Encephalopathy & 1.94 & $1.21-3.09$ & 0.006 \\
Previous PVT & 6.01 & $3.35-10.77$ & $<0.0001$ \\
Previous upper GI bleeding & 1.88 & $1.17-3.00$ & 0.008 \\
HCC presence & 2.57 & $1.69-3.92$ & $<0.0001$ \\
Diuretics use & 1.50 & $0.99-2.27$ & 0.055 \\
Panel B & & & \\
Previous PVT & 7.42 & $3.90-14.12$ & $<0.0001$ \\
Child-Pugh B + C & 2.72 & $1.75-4.23$ & $<0.0001$ \\
HCC presence & 2.14 & $1.35-3.39$ & 0.010 \\
Previous upper GI bleeding & 1.75 & $1.05-2.93$ & 0.030 \\
Age & 1.02 & $1.00-1.04$ & 0.012 \\
\hline
\end{tabular}

more frequent history of prior PVT. This finding is of interest as it shows that cirrhotic patients with PVT are at higher risk of recurrence and possibly should be treated to prevent it. So far, however, such perception as well as the need of treating cirrhotic PVT patients with anticoagulants seems to be weak; thus, $>50 \%$ of cirrhotic patients with PVT were not treated with anticoagulant despite some evidence of clinical benefit from the use of anti-thrombotic drugs in this specific setting $[24,25]$. This underuse is likely dependent on the persistent concept of "coagulopathy in cirrhosis", which may be a barrier against the use of anticoagulants in cirrhotic patients with PVT [26], the ongoing debate regarding the clinical significance of PVT and whether or not it is clinically significant or represents an epiphenomenon of advanced liver disease.

Another factor independently associated with PVT is upper GI bleeding, which is likely a mirror of the portal hypertension associated with PVT and, hence, reflects the already recognized higher risk of bleeding in cirrhotic patients with PVT [2, 21].
The study has limitations and implications. The crosssectional nature of the study does not allow prospectively analyzing PVT predictors and incidence in the cirrhotic population; the follow-up, currently ongoing in the PROLIVER study, will be useful to evaluate these issues. The study has been done in a Caucasian population; therefore, our findings cannot be extrapolated to other populations. The validation of the PVT using a CT scan would be useful, but was not requested by the protocol. However, the standardization of US parameters should guarantee the quality of the imaging data collection. The low rate of recruitment per center, despite no restrictive patient inclusion criteria, could be explained by the predominant involvement of an internal medicine services network. Patients with moderate to severe liver failure are at high risk of PVT and should be routinely screened for PVT even in the absence of specific symptoms, particularly in older patients or with a previous history of PVT. However, we did not investigate if the clinical history was complicated by deep venous thrombosis, which seems to complicate the clinical course of cirrhosis.

In conclusion, PVT is a frequent complication of cirrhosis, particularly in patients with moderate-severe liver failure. The significance of the condition remains a matter of debate, but in our opinion undertreatment of patients with PVT is a persistent matter of concern, which should be addressed by planning interventional trials with old or new oral anticoagulants.

\section{Compliance with ethical standards}

Conflict of interest The authors declare that they have no conflict of interest.

Statement of human and animal rights All procedures performed in studies involving human participants were in accordance with the ethical standards of the institutional and/or national research committee and with the 1964 Helsinki declaration and its later amendments or comparable ethical standards.

Informed consent Informed consent was obtained from all individual participants included in the study.

Financial support Nothing to declare. 


\section{PRO-LIVER Study Collaborators}

Palasciano Giuseppe, D'Alitto Felicia, Palmieri Vincenzo Ostilio, Santovito Daniela (Clinica Medica, UOC Medicina Interna Universitaria “A. Murri”), Di Michele Dario, Croce Giuseppe (U.O.C. di Medicina Interna - Ospedale "G.Mazzini" ASL Teramo), Sacerdoti David, Brocco Silvia, Fasolato Silvano, Cecchetto Lara, Bombonato Giancarlo (Department of Medicine, University of Padova, Padova, Italy), Bertoni Michele, Restuccia Tea (U.O.C. Medicina Interna 2, Nuovo Ospedale di Prato), Andreozzi Paola, Liguori Maria Livia (Centro di Medicina Predittiva, prevenzione e cura della malattie cronicodegenerative e controllo dei fattori di rischio, Azienda Policlinico Umberto I, Roma), Perticone Francesco, Caroleo Benedetto, Perticone Maria, Staltari Orietta (Department of Medical and Surgical Sciences, University of Catanzaro; UO Malattie Cardiovascolari Geriatriche, Policlinico Mater Domini di Catanzaro; Dipartimento di Medicina Sperimentale e Clinica, Università Magna Graecia di Catanzaro; Dipartimento Scienze della Salute, Università Magna Graecia di Catanzaro), Manfredini Roberto, De Giorgi Alfredo (UOC Clinica Medica, Azienda Ospedaliero Universitaria di Ferrara), Averna Maurizio, Giammanco Antonina (Dipartimento Biomedico di Medicina Interna e Specialistica, Università degli Studi di Palermo), Granito Alessandro, Pettinari Irene, Marinelli Sara, Bolondi Luigi (U.O. Medicina Interna, Dipartimento di Scienze Mediche e Chirurgiche,Università di Bologna), Falsetti Lorenzo, Salvi Aldo (Medicina Interna Generale e Subintensiva Ospedali Riuniti di Ancona), Durante-Mangoni Emanuele, Cesaro Flavio, Farinaro Vincenza, Ragone Enrico (Medicina Infettivologica e dei Trapianti, Seconda Università di Napoli), Morana Ignazio (U.O. Medicina Interna Area Critica, ARNAS "Garibaldi" Catania), Andriulli Angelo, Ippolito Antonio, Iacobellis Angelo, Niro Grazia, Merla Antonio (Gastroenterologia ed Endoscopia Digestiva-Casa Sollievo della Sofferenza -Ospedale-IRCCS San Giovanni Rotondo), Raimondo Giovanni, Maimone Sergio, Cacciola Irene, Varvara Doriana (Division of Clinical and Molecular Hepatology, Department of Internal Medicine University Hospital of Messina), Drenaggi Davide, Staffolani Silvia (Clinica Malattie Infettive Tropicali, Parassitologia, Epatiti croniche Azienda Ospedaliero Universitaria “Ospedali Riuniti”), Picardi Antonio, Vespasiani-Gentilucci Umberto, Galati Giovanni, Gallo Paolo (UOS Medicina Clinica, Epatologia Università Campus Bio-Medico), Davì Giovanni, Schiavone Cosima, Santilli Francesca, Tana Claudio (UO Patologia Medica, Ospedale Clinicizzato "SS. Annunziata" Chieti), Licata Anna, Soresi Maurizio (UOC di Medicina Interna ed Epatologia, DIBIMIS, Università di Palermo), Bianchi
Giovanni Battista, Carderi Isabella (UOC di Medicina Generale dell'Ospedale "SS. Capitanio e Gerosa" di Lovere), Pinto Antonio, Tuttolomondo Antonino (Unità Operativa Complessa di Medicina Interna e Cardioangiologia, Dipartimento Biomedico di Medicina Interna e Specialistica, Università degli Studi di Palermo), Ferrari Giovanni (U.O.C. di Medicina Interna, Ospedale "SS.Annunziata" di Varzi, A.O della Provincia di Pavia), Gresele Paolo, Fierro Tiziana, Morelli Olivia (S.S. di Diagnosi e Terapia delle Malattie Emorragiche e Laboratorio delle Malattie Tromboemboliche; S.C. Medicina Interna e Cardiovascolare, Dipartimento di Medicina; S.C. Gastroenterologia ed Epatologia, Università - Azienda Ospedaliera di Perugia), Laffi Giacomo, Romanelli Roberto Giulio, Arena Umberto, Stasi Cristina (Azienda Ospedaliero Universitaria Careggi, Unita' Operativa Complessa Medicina Interna ed Epatologia, Firenze), Gasbarrini Antonio, Gargovich Matteo, Zocco Maria Assunta, Riccardi Laura, Ainora Maria Elena (U.O.C. Medicina Interna e Gastroenterologia, Policlinico Gemelli, Roma), Capeci William, Martino Giuseppe Pio, Nobili Lorenzo (Clinica Medica Generale - AOU “Ospedali Riuniti" Ancona), Cavallo Maurizio (Medicina di Arzignano/Lonigo -ULSS 5 Ovest Vicentino), Frugiuele Pierluigi, Greco Antonio (Struttura Complessa di Medicina Interna e Reumatologia Azienda Ospedaliera di Cosenza), Pietrangelo Antonello, Ventura Paolo, Cuoghi Chiara, Marcacci Matteo (Divisione Medicina Interna 2 / CEMEF e Centro Dipartimentale di Ricerca Epatologica Avanzata "Mario Coppo", Azienda Ospedaliero-Universitaria Policlinico di Modena), Serviddio Gaetano, Vendemiale Gianluigi, Villani Rosanna, Gargano Ruggiero (Centro Universitario per la ricerca e cura delle malattie epatiche - Clinica Medica Universitaria, Foggia), Vidili Gianpaolo, Di Cesare Valentina, Masala Maristella, Delitala Giuseppe (Clinica Medica , Azienda Ospedaliero Universitaria Sassari), Invernizzi Pietro (Center for Autoimmune Liver Diseases, Humanitas Clinical and Research Center, Rozzano), Di Minno Giovanni, Tufano Antonella (Centro di Coordinamento Regionale per le Emocoagulopatie, AOU Federico II Napoli), Purrello Francesco, Privitera Graziella (Dipartimento di Medicina Clinica e Sperimentale Università di Catania, UOC di Medicina Interna Ospedale Garibaldi-Nesima, Catania), Forgione Alessandra, Curigliano Valentina (IRCCS San Raffaele Pisana Roma), Senzolo Marco, Rodríguez-Castro Kryssia Isabel (Unità Trapianto Multiviscerale, Gastroenterologia, Università Ospedale Di Padova), Giannelli Gianluigi (Dipartimenti delle Emergenze e dei Trapianti di Organo, Sezione di Medicina Interna, Allergologia ed Immunologia Clinica, Bari), Serra Carla (Ecografia diagnostica ed interventistica, Dipartimento delle Insufficienze d'organo e dei Trapianti, Policlinico S.Orsola-Malpighi, 
Bologna), Neri Sergio (UOC di Medicina Interna "A. Francaviglia", Policlinico Universitario di Catania), Pignataro Pietro (UOC I Medicina Interna, AOU Policlinico Vittorio Emanuele, Catania), Rizzetto Mario, Debernardi Venon Wilma (UOADU Gastroepatologia, Azienda Ospedaliera San Giovanni Battista Di Torino), Svegliati Baroni Gianluca (Clinica di Gastroenterologia, Ancona), Bergamaschi Gaetano, Masotti Michela, Costanzo Filippo $\left(\mathrm{I}^{\circ}\right.$ Clinica Medica, Fondazione IRCCS Policlinico San Matteo, University of Pavia, Italy), Angelico Francesco, Del Ben Maria, Napoleone Laura, Polimeni Licia, Raparelli Valeria, Talerico Giovanni, Proietti Marco, Romiti Giulio Francesco, Ruscio Eleonora, Toriello Filippo (I Clinica Medica, Sapienza University of Rome, Italy).

\section{References}

1. Tripodi A, Mannucci PM (2011) The coagulopathy of chronic liver disease. N Engl J Med 365:147-156

2. Ferro D, Angelico F, Caldwell SH, Violi F (2012) Bleeding and thrombosis in cirrhotic patients: what really matters? Dig Liver Dis 44:275-279

3. Fimognari FL, Violi F (2008) Portal vein thrombosis in liver cirrhosis. Intern Emerg Med 3:213-218

4. Tsochatzis EA, Senzolo M, Germani G, Gatt A, Burroughs AK (2010) Systematic review: portal vein thrombosis in cirrhosis. Aliment Pharmacol Ther 31:366-374

5. Yang ZJ, Costa KA, Novelli EM, Smith RE (2014) Venous thromboembolism in cirrhosis. Clin Appl Thromb Hemost 20:169-178

6. Northup PG, McMahon MM, Ruhl AP, Altschuler SE, VolkBednarz A, Caldwell SH et al (2006) Coagulopathy does not fully protect hospitalized cirrhosis patients from peripheral venous thromboembolism. Am J Gastroenterol 101:1524-1528

7. Aldawood A, Arabi Y, Aljumah A, Alsaadi A, Rishu A, Aldorzi $\mathrm{H}$ et al (2011) The incidence of venous thromboembolism and practice of deep venous thrombosis prophylaxis in hospitalized cirrhotic patients. Thromb J 9:1

8. Zocco MA, Di Stasio E, De Cristofaro R (2009) Thrombotic risk factors in patients with liver cirrhosis: correlation with MELD scoring system and portal vein thrombosis development. J Hepatol 51:682-689

9. Alkim H, Ayaz S, Sasmaz N, Oguz P, Sahin B (2012) Hemostatic abnormalities in cirrhosis and tumor-related portal vein thrombosis. Clin Appl Thromb Hemost 18:409-415

10. Amitrano L, Guardascione MA, Brancaccio V, Margaglione M, Manguso F, Iannaccone L et al (2004) Risk factors and clinical presentation of portal vein thrombosis in patients with liver cirrhosis. J Hepatol 40:736-741
11. Nery F, Chevret S, Condat B, de Raucourt E, Boudaoud L, Rautou PE et al (2015) Causes and consequences of portal vein thrombosis in 1,243 patients with cirrhosis: results of a longitudinal study. Hepatology 61:660-667

12. Pugh RN, Murray-Lyon IM, Dawson JL, Pietroni MC, Williams R (1973) Transection of the oesophagus for bleeding oesophageal varices. Br J Surg 60:646-649

13. Kamath PS, Wiesner RH, Malinchoc M, Kremers W, Therneau TM, Kosberg CL et al (2001) A model to predict survival in patients with end-stage liver disease. Hepatology 33:464-470

14. D'Amico G, Garcia-Tsao G, Abraldes J, Ginès P, Kim WR, Schepis F, et al (2005) Prognostic indicators in cirrhosis. In: de Franchis R (ed) Portal Hypertension IV. Proceedings of the Fourth Baveno International Consensus Workshop on Definitions, Methodology and Therapeutic Strategies. Blackwell, Oxford

15. Yerdel MA, Gunson B, Mirza D, Karayalçin K, Olliff S, Buckels $\mathrm{J}$ et al (2000) Portal vein thrombosis in adults undergoing liver transplantation: risk factors, screening, management, and outcome. Transplantation 69:1873-1881

16. Violi F, Ferro D, Basili S, Lionetti R, Rossi E, Merli M et al (1997) Ongoing prothrombotic state in the portal circulation of cirrhotic patients. Thromb Haemost 77:44-47

17. Basili S, Merli M, Ferro D, Lionetti R, Rossi E, Riggio O et al (1999) Clotting activation after transjugular intrahepatic portosystemic stent shunt. Thromb Haemost 81:711-714

18. Basili S, Raparelli V, Violi F (2010) The coagulopathy of chronic liver disease: is there a causal relationship with bleeding? Yes. Eur J Intern Med 21:62-64

19. Nolan JP (2010) The role of intestinal endotoxin in liver injury: a long and evolving history. Hepatology 52:1829-1835

20. Giannini EG, Stravitz RT, Caldwell SH (2015) Portal vein thrombosis and chronic liver disease progression: The closer you look the more you see. Hepatology. doi:10.1002/hep.27875

21. Violi F, Ferro D (2013) Clotting activation and hyperfibrinolysis in cirrhosis: implication for bleeding and thrombosis. Semin Thromb Hemost 39:426-433

22. Fimognari FL, De Santis A, Piccheri C, Moscatelli R, Gigliotti F, Vestri A et al (2005) Evaluation of D-dimer and factor VIII in cirrhotic patients with asymptomatic portal venous thrombosis. J Lab Clin Med 146:238-243

23. Violi F, Basili S, Raparelli V, Chowdary P, Gatt A, Burroughs AK (2011) Patients with liver cirrhosis suffer from primary haemostatic defects? Fact or fiction? J Hepatol 55:1415-1427

24. Villa E, Cammà C, Marietta M, Luongo M, Critelli R, Colopi S et al (2012) Enoxaparin prevents portal vein thrombosis and liver decompensation in patients with advanced cirrhosis. Gastroenterology 143:1253-1260

25. Lisman T, Kamphuisen PW, Northup PG, Porte RJ (2013) Established and new-generation antithrombotic drugs in patients with cirrhosis-possibilities and caveats. J Hepatol 59:358-366

26. Violi F (2015) Should the term coagulopathy in cirrhosis be abandoned? JAMA Intern Med 175:862-863 